\title{
Aperture Selection for ACO-OFDM in Free-Space Optical Turbulence Channel
}

\author{
Junyi Jiang, Peichang Zhang, Rong Zhang, Sheng Chen, Fellow, IEEE, and Lajos Hanzo, Fellow, IEEE
}

\begin{abstract}
We propose a novel aperture selection (ApS) scheme for asymmetrically clipped optical orthogonal frequency-division multiplexing (ACO-OFDM) based multiple transmit/receive aperture (MTRA) system communicating over a free-space optical (FSO) turbulence channel between a pair of cooperating base-stations in a vehicular communication system. Our proposed ApS scheme is capable of significantly improving both the system's achievable diversity gain, its bit error ratio as well as its throughput, while imposing a substantially reduced power consumption and hardware complexity. Our results demonstrate that the proposed system's performance was improved more significantly under strongly turbulent channel conditions.
\end{abstract}

Index Terms-Free-space optical turbulence channel, asymmetrically clipped optical orthogonal frequencydivision multiplexing, multiple transmit/receive aperture, aperture selection

\section{INTRODUCTION}

$\mathbf{F}$ REE-space optical (FSO) links [1], [2] provide an attractive design alternative for base station-base station (BS-BS) in cooperative multicell (COMP) scenarios [3], [4] to the classic radio-frequency $(\mathrm{RF})$ microwave links, where the turbulent outdoor channel suffers from adverse propagation conditions. They also offer a design alternative for the extremely challenginga ircraft-to-aircraft and aircraftto-satellite vehicular communications scenarios [5]-[7], with the goal of providing sophisticate wireless multimedia service, and Internet access above the clouds [8]. Whilst at the time of writing, most of the solutions considered in the literature rely on radio communications with satellites [9][16], we believe that FSO solution may also be developed for this emerging vehicular communications scenario. Therefore, multiple-transmit-and receive aperture (MTRA) aided techniques, may be invoked both for increasing the system's throughput as well as for acquiring a diversity gain [17]. Hence MTRA techniques are capable of offering an improved bit error ratio (BER) by mitigating the above-mentioned detrimental propagation effects of FSO communications at the expense of increasing the hardware complexity and power consumption.

ACO-OFDM has been used in numerous systems designed for indoor VLC [18]-[20], despite its factor four throughput reduction imposed by its Hermitian symmetry, which has to be satisfied for the sake of generating a real and positivevalued optical modulated signals. Even though the indoor propagation of light only imposes mild dispersion in moderately sized rooms, ACO-OFDM has the innate ability to eliminate the dispersion imposed by multipath reflections, because each subcarrier transmits a low-rate sequence that remains unaffected by dispersion. In contrast to the small

Copyright (c) 2015 IEEE. Personal use of this material is permitted. However, permission to use this material for any other purposes must be obtained from the IEEE by sending a request to pubspermissions@ieee.org.

The authors are with Electronics and Computer Science, University of Southampton, Southampton SO17 1BJ, U.K. (E-mails: \{jj4e09, pz3g09, rz, sqc, lh\}@ecs.soton.ac.uk).

The financial supports of RC-UK under the auspices of the IndiaUK Advanced Technology Centre and of EU Concerto project, as well as the European Research Council's Advanced Fellow Grant are gratefully acknowledged. indoor attocells, FSO systems communicate over much larger distances and encounter commensurately longer CIRs, which is beneficially counteracted by the anti-dispersion capability of ACO-OFDM, as argued in [21]-[24].

This situation is similar to that of classic RF MIMO systems, which require multiple $\mathrm{RF}$ chains. Due to the systemlevel similarity between RF MIMOs and FSO MTRAs, the philosophy of antenna selection (AS) techniques originally conceived for conventional RF MIMOs [25]-[27] may also be applied to FSO MTRAs, leading to the concept of 'aperture selection' (ApS) [28]-[31], which inherits the low-cost and low-complexity benefits from the classic RF AS. In [28], the concept of transmit selection is firstly introduced to FSO communication, and it was proved to be capable of achieving full diversity, whilst the selection at both transmit and receive sides for multi-element FSO is comprehensively studied in [29], in [30] a practical application of ApS in mobile FSO nodes is exhibited, furthermore, as an extension of $\mathrm{ApS}$, the relay selection based on channel information is described in [31]. Hence, in FSO MTRA systems, given the fixed number of optical chains ${ }^{1}$, employing ApS is capable of significantly improving the system's achievable performance. Furthermore, as the average channel variance increases with the turbulence level of the FSO channel, adopting ApS in FSO MTRAs become beneficial, since the specific links exhibiting the highest power will be activated.

Against the above background, in this paper, we propose a novel ACO-OFDM [18]-[20] based FSO MTRA system relying on joint ApS for transmission over FSO turbulence channels ${ }^{2}$, which employs joint maximum likelihood (ML) hard detection (HD) for uncoded systems and joint maximum a posteriori probability (MAP) soft detection (SD) for forward error correction (FEC) coded systems. Our contributions are as follows.

- We construct a novel ACO-OFDM based MTRA system and investigate its performance using different detection schemes. We will demonstrate that the resultant BER curves corresponding to different levels of turbulence reveal an intriguing curve crossing phenomenon.

- We design a novel ApS scheme based on exploiting the FSO channel characteristics for assisting our ACOOFDM based MTRA system, which is capable of significantly improving both the achievable BER and throughput, while imposing a reduced power consumption and hardware complexity. We will show that the performance gain of our ApS scheme becomes more significant under hostile turbulence channel conditions.

This paper is organized as follows. Section II introduces our ACO-OFDM based MTRA optical wireless (OW) system designed for the FSO turbulence channels, while Section III details the joint ApS scheme proposed for effectively exploiting the system's available optical chains. Both BER per-

${ }^{1}$ In this case, we define an entire set of electronic hardware components at transmit/receive end, including electronic-to-optical conversion or optical-to-electronic conversion, as an optical chain.

${ }^{2}$ Please note that, the proposed ApS scheme is applicable to any arbitrary OFDM-based system. However, for the simplicity of exposition, but without any loss of generality, we only use ACOOFDM in this paper. 
formance and extrinsic information transfer (EXIT) chartbased throughput analysis of our system are presented in Section IV. Our conclusions are summarized in Section V.

Throughout our discussions, ()$^{\mathrm{T}}$ and ()$^{\mathrm{H}}$ denote the transpose and conjugate transpose operators, respectively, while ()$^{-1}$ and ()$^{*}$ stand for the inverse and conjugate operations, respectively. $I$ denotes the identity matrix with an appropriate dimension. Under the MTRA context, we have the $N_{t}$ transmit apertures at transmitter and the $N_{r}$ receive apertures at receiver. Additionally, $\mathbb{T}(\cdot)$ denotes the composite SD-decoding transfer function.

\section{ACO-OFDM BASED MTRA SYSTEM}

\section{A. $A C O-O F D M$}

The ACO-OFDM input vector of the $m$ th MTRA substream $0 \leq m \leq M-1$ is constituted by the $N_{F} / 4$ frequency domain (FD) data symbols given by $\boldsymbol{S}_{A C O}^{(m)}=$ $\left[S_{0}^{(m)} S_{1}^{(m)} \cdots S_{N_{F} / 4-1}^{(m)}\right]^{\mathrm{T}}$, where $M$ is the total number of optical chains and $N_{F}$ represents the length of an OFDM symbols. Quadrature amplitude modulation (QAM) signalling is employed. ACO-OFDM only uses the odd-indexed subcarriers to carry data while assigning ' $0 \mathrm{~s}$ ' to the evenindexed subcarriers as well as simultaneously adopting a Hermitian-symmetric symbol arrangement. Thus $\boldsymbol{S}_{A C O}^{(m)}$ is first expanded into the form

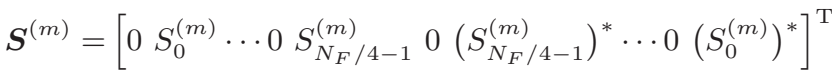

$$
\begin{aligned}
& =\left[\begin{array}{lll}
\bar{S}_{0}^{(m)} & \bar{S}_{1}^{(m)} \cdots \bar{S}_{N_{F}-1}^{(m)}
\end{array}\right]^{\mathrm{T}} .
\end{aligned}
$$

By defining the $N_{F}$-point inverse fast Fourier transform (IFFT) matrix as

$$
\boldsymbol{F}=\frac{1}{\sqrt{N_{F}}}\left[\begin{array}{cccc}
1 & 1 & \cdots & 1 \\
1 & e^{\mathrm{j} 2 \pi / N_{F}} & \cdots & e^{\mathrm{j} 2 \pi\left(N_{F}-1\right) / N_{F}} \\
\vdots & \vdots & \ddots & \vdots \\
1 & e^{\mathrm{j} 2 \pi\left(N_{F}-1\right) / N_{F}} & \cdots & e^{\mathrm{j} 2 \pi\left(N_{F}-1\right)^{2} / N_{F}}
\end{array}\right],
$$

where $\mathrm{j}=\sqrt{-1}$, the time-domain (TD) OFDM symbol vector is given by

$$
\boldsymbol{s}^{(m)}=\left[\begin{array}{lll}
s_{0}^{(m)} & s_{1}^{(m)} \cdots s_{N_{F}-1}^{(m)}
\end{array}\right]^{\mathrm{T}}=\boldsymbol{F} \boldsymbol{S}^{(m)} .
$$

All the negative-valued elements in $\boldsymbol{s}^{(m)}$ are forced to become zero by the clipping operation. As a benefit of this ACOOFDM modulation arrangement, the useful odd-indexed subcarriers are protected from the detrimental effects of clipping noise. Consequently, both the real and imaginary information of the original FD data in $S^{(m)}$, which are spread along all the TD OFDM symbols, are well preserved. After concatenating the $N_{c p}$-length cyclic prefix (CP) to $\boldsymbol{s}^{(m)}$, the resultant $\left(N_{F}+N_{c p}\right)$-length vector $\boldsymbol{s}_{o}^{(m)}$ is converted into the optical signal by the electronic to optical (E/O) converter for transmission.

At the receiver end, after the optical to electronic $(\mathrm{O} / \mathrm{E})$ conversion and the removal of the $\mathrm{CP}$, the $N_{F}$-length TD signal vector received by the $n$th receiver chain is given by

$$
\boldsymbol{y}^{(n)}=R \cdot \overline{\mathbf{H}}^{(n, m)} \boldsymbol{s}_{o}^{(m)}+\mathbf{v}^{(n, m)},
$$

where $\overline{\mathbf{H}}^{(n, m)}$ represents the $N_{F} \times\left(N_{F}+N_{c p}\right)$-element linear convolution channel matrix between the $m$ th transmitter and the $n$ the receiver, while $\mathbf{v}^{(n, m)}$ denotes the TD additive white Gaussian noise (AWGN) vector imposed on the channel linking the $m$ th transmit aperture (TAp) to the $n$th receive aperture (RAp), whose elements have zero mean and a variance of $\sigma^{2}$, while $R$ [W/A] is the responsivity of the photodiode. Eq. (4) is equivalent to

$$
\boldsymbol{y}^{(n)}=R \cdot \mathbf{H}^{(n, m)} \boldsymbol{s}^{(m)}+\mathbf{v}^{(n, m)},
$$

where $\mathbf{H}^{(n, m)}$ is the circulant convolution channel matrix of $\left(N_{F} \times N_{F}\right)$ elements [32]

$$
\mathbf{H}^{(n, m)}=
$$

$$
\left[\begin{array}{ccccccc}
h_{0} & 0 & \cdots & 0 & \cdots & h_{2} & h_{1} \\
h_{1} & h_{0} & \cdots & \vdots & \cdots & h_{3} & h_{2} \\
\vdots & \vdots & \ddots & 0 & \ddots & \vdots & \vdots \\
h_{\mathrm{N}_{\mathrm{cp}}} & h_{\mathrm{N}_{\mathrm{cp}}-1} & \cdots & h_{0} & \cdots & h_{\mathrm{N}_{\mathrm{cp}}-2} & h_{\mathrm{N}_{\mathrm{cp}}-1} \\
0 & h_{\mathrm{N}_{\mathrm{cp}}} & \cdots & \vdots & \ddots & \vdots & \vdots \\
\vdots & \vdots & \ddots & h_{\mathrm{N}_{\mathrm{cp}}-1} & \cdots & h_{0} & 0 \\
0 & 0 & \cdots & h_{\mathrm{N}_{\mathrm{cp}}} & \cdots & h_{1} & h_{0}
\end{array}\right]
$$

with each channel impulse response represented by independent random variables obeying the Gamma-Gamma distribution. Furthermore, $R \cdot \mathbf{H}^{(n, m)}=\boldsymbol{F} \boldsymbol{\Lambda}^{(n, m)} \boldsymbol{F}^{\mathrm{H}}$, in which $\boldsymbol{\Lambda}^{(n, m)}$ is the $\left(N_{F} \times N_{F}\right)$-element diagonal matrix with eigenvalues of $\mathbf{H}^{(n, m)}$ at its diagonal entries. By applying the fast Fourier transform (FFT) based OFDM demodulation to $y^{n}$, we obtain the received $N_{F}$-length FD symbol vector

$$
\boldsymbol{Y}_{o}^{(n)}=\boldsymbol{F}^{\mathrm{H}} \boldsymbol{y}^{(n)}=\boldsymbol{\Lambda}^{(n, m)} \boldsymbol{S}^{(m)}+\boldsymbol{V}^{(n, m)},
$$

where the FD channel noise vector $\boldsymbol{V}^{(n, m)}=\boldsymbol{F}^{\mathrm{H}} \mathbf{v}^{(n, m)}$ also obeys the same AWGN distribution as $\mathbf{v}^{(n, m)}$.

Upon collecting all the odd-indexed elements in the first half of $\boldsymbol{Y}_{o}^{(n)}$ and arranging them in the $N_{F} / 4$-length vector $\boldsymbol{Y}_{A C O}^{(n)}$, we have

$$
\boldsymbol{Y}_{A C O}^{(n)}=\boldsymbol{\Lambda}_{A C O}^{(n, m)} \boldsymbol{S}_{A C O}^{(m)}+\boldsymbol{V}_{A C O}^{(n, m)}
$$

where $\Lambda_{A C O}^{(n, m)}$ is the $\left(N_{F} / 4\right) \times\left(N_{F} / 4\right)$-element diagonal matrix representing the first $N_{F} / 4$ odd-indexed elements extracted from the diagonal of $\boldsymbol{\Lambda}^{(n, m)}$, which $\boldsymbol{V}_{A C O}^{(n, m)}$ contains the first $N_{F} / 4$ odd-indexed elements of $\boldsymbol{V}^{(n, m)}$. Clearly, $\boldsymbol{Y}_{A C O}^{(n)}$ constitutes sufficient statistics for estimating $\boldsymbol{S}_{A C O}^{(m)}$.

\section{B. Channel Model}

FSO turbulent channels impose numerous detrimental effects, such as pathloss-induced attenuation and scintillation. These adverse effects can be modelled by a Gamma-Gamma distribution [33], where irradiance is characterized by the product of a pair of independent Gamma-distributed random processes. The probability density function (PDF) of the channel impulse response (CIR) is given by [33]

$$
p_{G G}(\gamma)=\frac{2(\alpha \beta)^{\frac{\alpha+\beta}{2}}}{\Gamma(\alpha) \Gamma(\beta)} \gamma^{\frac{\alpha+\beta}{2}-1} \cdot K_{\alpha-\beta}(2 \sqrt{\alpha \beta \cdot \gamma}),
$$

where $\Gamma(\cdot)$ denotes the Gamma function, $K_{\nu}(\cdot)$ is the modified Bessel function of the second kind, while $\alpha$ and $\beta$ are the pair of parameters controlling the two Gamma functions.

Specifically, $\alpha$ determines the large scale scattering caused by refractive effects, which results in beam direction deviation, and it is expressed by [33]

$$
\alpha=\left\{\exp \left[\frac{0.49 \sigma_{r}^{2}}{\left(1+0.18 d^{2}+0.56 \sigma_{r}^{12 / 5}\right)^{7 / 6}}\right]-1\right\}^{-1},
$$


whilst $\beta$ is attributed to the diffraction that leads to beam spreading and irradiance fluctuation, which is given by [33]

$$
\beta=\left\{\exp \left[\frac{0.51 \sigma_{r}^{2}\left(1+0.69 \sigma_{r}^{12 / 5}\right)^{-5 / 6}}{\left(1+0.9 d^{2}+0.62 \sigma_{r}^{12 / 5}\right)^{5 / 6}}\right]-1\right\}^{-1} .
$$

In Eqs. (10) and (11), $d=\sqrt{k D^{2} / 4 L}$, where $k=2 \pi / \lambda$ denotes the number of optical waves with $\lambda$ being the wavelength, $L$ indicates the physical link distance between the TAp and the RAp, while $D$ is the diameter of the RAp. Furthermore, $\sigma_{r}^{2}$ is known as the Rytov variance, which directly relates to the optical variance of the FSO channel, and it is given by

$$
\sigma_{r}^{2}=0.5 C_{n}^{2} k^{7 / 6} L^{11 / 6},
$$

where $C_{n}^{2}$ is the refractive index that determines the level of turbulence.

It is worth emphasizing that although $C_{n}^{2}$ plays a similar role in the Gamma-Gamma channel to that of the normalized Doppler frequency $f_{d}$ in the classic RF Rayleigh fading channel, they also have significant differences, as listed in Table I. Explicitly, in Table I, we can see not only the similar roles played by $f_{d}$ and $C_{n}^{2}$ in the corresponding channels, but also their differences. Specifically, unlike the RF Rayleigh fading channel, whose variance does not vary with $f_{d}$, the variance of the FSO turbulence channel varies with $C_{n}^{2}$. This points to the potential of acquiring a considerable diversity gain by exploiting ApS techniques in FSO MTRA communication, especially under stronger turbulence. This is because ApS is capable of choosing the channels with higher gains, leading to an enhanced performance in FSO MTRA communication.

\section{System Structure}

Our proposed ACO-OFDM based MTRA OW system is depicted in Fig. 1, where all the component blocks on the left hand side of the vertical dashed line represent the electronic hardware and circuits, while on the right hand side of this dashed line, after the $\mathrm{E} / \mathrm{O}$ and $\mathrm{O} / \mathrm{E}$ conversions, the signals are transmitted to and received from the corresponding apertures. Additionally, the FEC encoding block and the iterative decoding block are only related to the coded system relying on soft detection and decoding. The numbers of the available optical chains at the transmitter and receiver are $M$ and $N$, respectively. Generally speaking, we aim for $M<N_{t}$ and $N<N_{r}$, where the joint ApS module of Fig. 1 selects $M$ transmit apertures from the total of $N_{t}$ TAps and $N$ receive apertures from the total of $N_{r}$ RAps to form an actual $(M \times N)$-element MTRA OW system for communication. In the special case of $M=N_{t}$ and $N=N_{r}$, this joint ApS module is not required. For the time being, we assume $M=N_{t}$ and $N=N_{r}$ since this is sufficient for us to describe all the components of the system illustrated in Fig. 1, except for the joint ApS module. Furthermore, the system of Fig. 1 is capable of adopting any MTRA scheme. Without loss of generality, in this paper we will mainly consider the spacetime block code (STBC) philosophy [34].

1) Uncoded joint ML-based HD system: As shown in Fig. 1, the original information bits, denoted as $b$, are firstly modulated into the $N_{F} / 4$-length QAM symbol vector denoted by $\boldsymbol{X}=\left[\begin{array}{ll}X_{0} & X_{1} \cdots X_{N_{F} / 4-1}\end{array}\right]^{\mathrm{T}}$, which is then mapped onto the $M$ sub-streams of $\boldsymbol{S}_{A C O}^{(m)}$ for $0 \leq m \leq M-1$, according to the STBC MTRA scheme:

$$
\boldsymbol{S}_{A C O}=\mathcal{F}_{\mathrm{STBC}}(\boldsymbol{X}),
$$

relying on the STBC mapping $\mathcal{F}_{\mathrm{STBC}}(\cdot)$. Taking the G2 STBC scheme $(M=2)$ as an example, the two rows of $\boldsymbol{S}_{A C O}$ are given by $S_{A C O}^{(0)}=\frac{1}{\sqrt{M}}\left[X_{0}-X_{1}^{*} \cdots X_{N_{F} / 4-2}-X_{N_{F} / 4-1}^{*}\right]^{\mathrm{T}}$ and $\boldsymbol{S}_{A C O}^{(1)}=\frac{1}{\sqrt{M}}\left[X_{1}-X_{0}^{*} \cdots X_{N_{F} / 4-1}-X_{N_{F} / 4-2}^{*}\right]^{\mathrm{T}}$. Each $\boldsymbol{S}_{A C O}^{(m)}$ is processed by an ACO-OFDM modulator to generate the TD unipolar signal vector $\boldsymbol{s}^{(m)}$, as detailed in Subsection II-A. After optical intensity modulation, $\boldsymbol{s}^{(m)}$ is launched through the $m$ th TAp associated with the $m$ th optical chain of the transmitter.

The $M$ signal sub-streams are transmitted over the $(N \times$ $M$ )-element MTRA FSO turbulence channel, having the characteristics described in Subsection II-B. At the receiver, after $\mathrm{O} / \mathrm{E}$, the $N$ received TD signal sub-streams $\boldsymbol{y}^{(n)}$ for $0 \leq n \leq N-1$ are passed through the ACO-OFDM demodulator to extract the useful complex-valued sequences $\boldsymbol{Y}_{A C O}^{(n)}$ for $0 \leq n \leq N-1$. Assuming the perfect knowledge of the CSI is known at the receiver, the joint ML detection of $\boldsymbol{X}$ can be carried out given $\boldsymbol{Y}_{A C O}^{(n)}$ for $0 \leq n \leq N-1$, which is denoted as $\widehat{\boldsymbol{X}}$. Finally, the estimate of the transmitted binary bit block $\boldsymbol{b}$, denoted as $\widehat{b}$, can be obtained by QAM demapping of the ML estimate $\widehat{\boldsymbol{X}}$.

More specifically, upon collecting all the received $\boldsymbol{Y}_{A C O}^{(n)}$ for $0 \leq n \leq N-1$ in the $\left(N \cdot N_{F} / 4\right)$-length vector we have: $\boldsymbol{Y}_{A C O}=\left[\left(\boldsymbol{Y}_{A C O}^{(0)}\right)^{\mathrm{T}}\left(\boldsymbol{Y}_{A C O}^{(1)}\right)^{\mathrm{T}} \cdots\left(\boldsymbol{Y}_{A C O}^{(N-1)}\right)^{\mathrm{T}}\right]^{\mathrm{T}}$. Then, according to Eq. (8), $\boldsymbol{Y}_{A C O}$ can be expressed as

$$
\boldsymbol{Y}_{A C O}=\boldsymbol{\Lambda}_{A C O} \boldsymbol{S}_{A C O}+\boldsymbol{V}_{A C O},
$$

in which $\boldsymbol{V}_{A C O}=\left[\left(\boldsymbol{V}_{A C O}^{(0)}\right)^{\mathrm{T}}\left(\boldsymbol{V}_{A C O}^{(1)}\right)^{\mathrm{T}} \cdots\left(\boldsymbol{V}_{A C O}^{(N-1)}\right)^{\mathrm{T}}\right]^{\mathrm{T}}$ with $\boldsymbol{V}_{A C O}^{(n)}=\sum_{m=0}^{M-1} \boldsymbol{V}_{A C O}^{(n, m)}$, while $\boldsymbol{S}_{A C O}=$ $\left[\left(\boldsymbol{S}_{A C O}^{(0)}\right)^{\mathrm{T}}\left(\boldsymbol{S}_{A C O}^{(1)}\right)^{\mathrm{T}} \cdots\left(\boldsymbol{S}_{A C O}^{(M-1)}\right)^{\mathrm{T}}\right]^{\mathrm{T}}$ and

$$
\boldsymbol{\Lambda}_{A C O}=\left[\begin{array}{cccc}
\boldsymbol{\Lambda}_{A C O}^{(0,0)} & \boldsymbol{\Lambda}_{A C O}^{(0,1)} & \cdots & \boldsymbol{\Lambda}_{A C O}^{(0, M-1)} \\
\boldsymbol{\Lambda}_{A C O}^{(1,0)} & \boldsymbol{\Lambda}_{A C O}^{(1,1)} & \cdots & \boldsymbol{\Lambda}_{A C O}^{(1, M-1)} \\
\vdots & \vdots & \vdots & \vdots \\
\boldsymbol{\Lambda}_{A C O}^{(N-1,0)} & \boldsymbol{\Lambda}_{A C O}^{(N-1,1)} & \cdots & \boldsymbol{\Lambda}_{A C O}^{(N-1, M-1)}
\end{array}\right]
$$

Therefore, the ML estimate $\widehat{\boldsymbol{X}}$ is the solution of the following optimization problem

$$
\widehat{\boldsymbol{X}}=\arg \underset{\widetilde{\boldsymbol{X}} \in \mathbb{X}}{\min }\left\|\boldsymbol{Y}_{A C O}-\boldsymbol{\Lambda}_{A C O} \mathcal{F}_{\mathrm{STBC}}(\widetilde{\boldsymbol{X}})\right\|^{2},
$$

where $\mathbb{X}$ denotes the feasible set of $\boldsymbol{X}$, i.e. $\boldsymbol{X}$ assumes a value from the set $\mathbb{X}$, and the size of $\mathbb{X}$ is $\zeta^{N_{F} / 4}$ for the $\zeta$-QAM signalling.

Note that there is a total of $\left(N_{r} \times N_{t}\right)$ FSO turbulence channels and the overall MTRA channel matrix can be expressed as:

$$
\mathbf{H}=\left[\begin{array}{cccc}
\mathbf{H}^{(0,0)} & \mathbf{H}^{(0,1)} & \cdots & \mathbf{H}^{\left(0, N_{t}-1\right)} \\
\mathbf{H}^{(1,0)} & \mathbf{H}^{(1,1)} & \cdots & \mathbf{H}^{\left(1, N_{t}-1\right)} \\
\vdots & \vdots & \vdots & \vdots \\
\mathbf{H}^{\left(N_{r}-1,0\right)} & \mathbf{H}^{\left(N_{r}-1,1\right)} & \cdots & \mathbf{H}^{\left(N_{r}-1, N_{t}-1\right)}
\end{array}\right]
$$

According to Eq. (5), the corresponding overall MTRA channel model is then given by

$$
\boldsymbol{y}=R \cdot \mathbf{H} \boldsymbol{s}+\mathbf{V},
$$

where we have $\boldsymbol{y}=\left[\left(\boldsymbol{y}^{(0)}\right)^{\mathrm{T}}\left(\boldsymbol{y}^{(1)}\right)^{\mathrm{T}} \cdots\left(\boldsymbol{y}^{\left(N_{r}-1\right)}\right)^{\mathrm{T}}\right]^{\mathrm{T}}$, and $\boldsymbol{s}=\left[\left(\boldsymbol{s}^{(0)}\right)^{\mathrm{T}}\left(\boldsymbol{s}^{(1)}\right)^{\mathrm{T}} \cdots\left(\boldsymbol{s}^{\left(N_{t}-1\right)}\right)^{\mathrm{T}}\right]^{\mathrm{T}}$, while the noise ma- 
TABLE I

A Comparison between Rayleigh Fading Channel and FSO Turbulence Channel

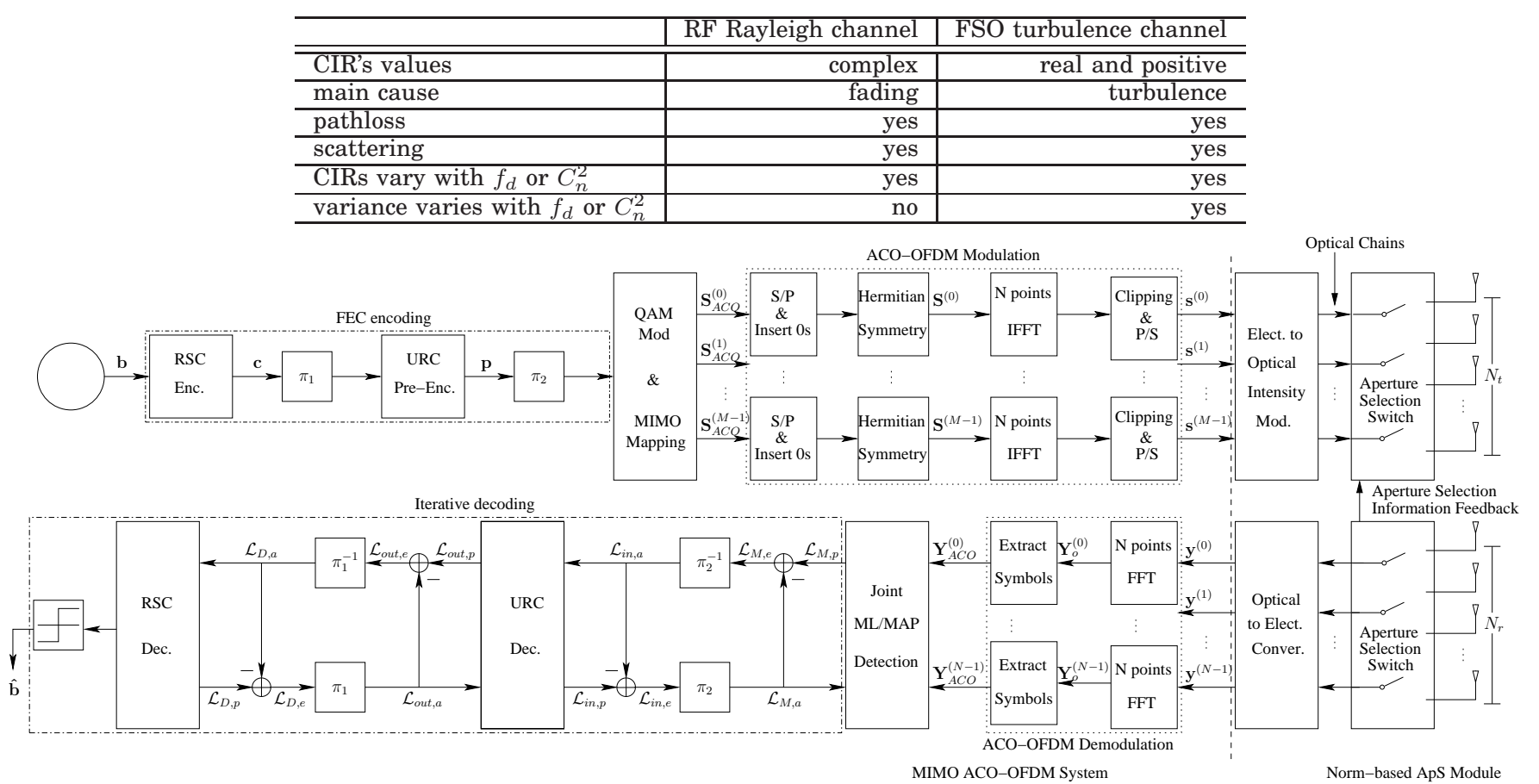

Fig. 1. Schematic of the proposed ACO-OFDM based MTRA system with the aid of the joint ApS, where $M$ and $N$ are the numbers of optical chains at the transmitter and receiver, respectively, while $N_{t}$ and $N_{r}$ are the numbers of transmit and receive apertures, respectively. In general, we have $M<N_{t}$ and $N<N_{r}$.

$\operatorname{trix} \mathbf{V}=\left[\left(\mathbf{V}^{(0)}\right)^{\mathrm{T}}\left(\mathbf{V}^{(1)}\right)^{\mathrm{T}} \cdots\left(\mathbf{V}^{\left(N_{r}-1\right)}\right)^{\mathrm{T}}\right]^{\mathrm{T}}$ cc $\mathbf{V}^{(n)}=$ $\sum_{m=0}^{N_{t}-1} \mathbf{V}^{(n, m)}, 0 \leq n \leq N_{r}-1$. To support such a large-

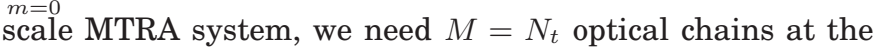
transmitter and $N=N_{r}$ optical chains at the receiver, which is costly.

2) Three-stage iterative joint MAP-based SD system: The information bit sequence $\boldsymbol{b}$ is first channel encoded by a half-rate recursive systematic code (RSC) based outer encoder, yielding the coded bit sequence $c$, which is then bitinterleaved by $\pi_{1}$ of Fig. 1. Furthermore, we invoke a unitrate code (URC) as the inner encoder, which is associated with the bit-interleaver $\pi_{2}$ of Fig. 1 . The explicit benefit of incorporating a low-complexity memory-1 URC is that it allows the system to beneficially spread the extrinsic information across the iterative decoder components without increasing its delay and, as a result, a vanishingly low BER is attainable [35]-[37]. Following the two-stage encoding process, the coded bits traverse through the same QAM modulator and STBC MTRA mapper as described in Subsection II-C1.

The corresponding three-stage turbo decoder of the receiver is also depicted in Fig. 1, which consists of a joint MAP-based SD, a URC decoder and a RSC decoder. More explicitly, the composite inner decoder is formed by the combined joint MAP-based SD and the URC decoder, where the associated a priori information and extrinsic information are firstly interleaved and exchanged $I_{\text {inner }}$ times. The outer decoder is constituted by the RSC decoder, where the information gleaned from the inner decoder is iteratively exchanged $I_{\text {outer }}$ times. The final HD is then carried out by the RSC decoder to produce the estimate $\widehat{b}$ of the transmitted information bit block $\boldsymbol{b}$.

For ease of explanation, the information exchanged be- tween the decoder components, in terms of log likelihood ratios (LLRs) [37] as depicted in Fig. 1, are first defined.

- $\mathcal{L}_{M, p}, \mathcal{L}_{M, a}, \mathcal{L}_{M, e}$ : the a posteriori, a priori and extrinsic LLRs, respectively, associated with the joint MAP-based SD block, which are detailed in [35], [38].

- $\mathcal{L}_{i n, p}, \mathcal{L}_{i n, a}, \mathcal{L}_{i n, e}$ : the a posteriori, a priori and extrinsic LLRs, respectively, associated with the URC decoder block.

- $\mathcal{L}_{\text {out }, p}, \mathcal{L}_{\text {out }, a}, \mathcal{L}_{\text {out }, e}$ : the a posteriori, a priori and extrinsic LLRs, respectively, associated with the composite inner decoder block.

- $\mathcal{L}_{D, p}, \mathcal{L}_{D, a}, \mathcal{L}_{D, e}$ : the a posteriori, a priori and extrinsic LLRs, respectively, associated with the outer RSC decoder block.

Since the $\zeta$-QAM scheme maps a set of $\eta=\log _{2} \zeta$ consecutive incoming bits onto a symbol value, we consider a set of $\boldsymbol{p}_{(1: \eta)}=\left\{p_{1}, p_{2}, \cdots, p_{\eta}\right\}$ consecutive bits without loss of generality. For the $v$ th bit of $\boldsymbol{p}_{(1: \eta)}$, its bit-wise $a$ posteriori LLR can be derived by the classic Max-Log approximation [35] as

$$
\begin{aligned}
\mathcal{L}_{M, p}\left(p_{v}\right) & =\mathcal{L}_{M, a}\left(p_{v}\right)+ \\
& \max _{\widetilde{\boldsymbol{X}} \in \mathbb{X}_{1}^{p_{v}}}\left\{-\left\|\boldsymbol{Y}_{A C O}-\boldsymbol{\Lambda}_{A C O} \mathcal{F}_{\mathrm{STBC}}(\widetilde{\boldsymbol{X}})\right\|^{2} / 2 \sigma^{2}+A\right\} \\
& -\max _{\widetilde{\boldsymbol{X}} \in \mathbb{X}_{0}^{p_{v}}}\left\{\left\|\boldsymbol{Y}_{A C O}-\boldsymbol{\Lambda}_{A C O} \mathcal{F}_{\mathrm{STBC}}(\widetilde{\boldsymbol{X}})\right\|^{2} / 2 \sigma^{2}+A\right\},
\end{aligned}
$$

where we have $A=\sum_{\tau=1, \tau \neq v}^{\eta} p_{v} \mathcal{L}_{M, a}\left(p_{\tau}\right)$, while $\mathbb{X}_{0}^{p_{v}}=$ $\left\{\widetilde{\boldsymbol{X}} \in \mathbb{X} \mid p_{v}=0\right\}$ and $\mathbb{X}_{1}^{p_{v}}=\left\{\widetilde{\boldsymbol{X}} \in \mathbb{X} \mid p_{v}=1\right\}$. The extrinsic LLRs $\mathcal{L}_{M, e}$ gleaned from the joint MAP-based SD block are deinterleaved and then they are fed as the a priori LLRs $\mathcal{L}_{i n, a}$ into the URC pre-decoder of Fig. 1, which in turn calcu- 
lates its a posteriori LLRs $\mathcal{L}_{\text {in,p }}$. Then the updated extrinsic LLRs $\mathcal{L}_{i n, e}$ are fed back and re-interleaved, before being processed as the a priori LLRs $\mathcal{L}_{M, a}$ by the SD block in the next inner iteration [35]-[37]. After the convergence of the inner iteration process after $I_{\text {in }}$ iterations, the outer iteration follows a similar procedure, which involves exchanging the corresponding extrinsic LLRs between the URC decoder and the RSC decoder, until the predefined stopping criterion, specified by the number of outer iterations $I_{\text {out }}$, is met.

\section{The Proposed Joint Aperture Selection SCHEME}

In practice, the number of available optical chains is limited, and typically we have $M \ll N_{t}$ and $N \ll N_{r}$. In order to most efficiently exploit the available optical chains, it is highly desirable to select the most appropriate subset of the $N \times M$ FSO links from the full set of the $N_{r} \times N_{t}$ channels for the ACO-OFDM based MTRA system having only $M$ optical chains at the transmitter and $N$ optical chains at the receiver. In other words, the task is to select $M$ appropriate TAps as well as $N$ appropriate RAps from the total of $N_{t}$ TAps and the total of $N_{r}$ RAps, respectively, in order to form the most beneficial $(N \times M)$-element subset MTRA channel matrix for actual communication. The ApS module of Fig. 1 is responsible for carrying out this task, and we adopt a lowcomplexity yet efficient ApS scheme.

A generic $(N \times M)$-element subset MTRA channel matrix $\mathbf{H}_{\text {sub }} \subset \mathbf{H}$, where $\mathbf{H}$ is the full $\left(N_{r} \times N_{t}\right)$ MTRA channel matrix of Eq. (17), can be formulated as:

$$
\mathbf{H}_{\text {sub }}=\left[\begin{array}{cccc}
\mathbf{H}^{\left(i_{0}, j_{0}\right)} & \mathbf{H}^{\left(i_{0}, j_{1}\right)} & \ldots & \mathbf{H}^{\left(i_{0}, j_{M-1}\right)} \\
\mathbf{H}^{\left(i_{1}, j_{0}\right)} & \mathbf{H}^{\left(i_{1}, j_{1}\right)} & \cdots & \mathbf{H}^{\left(i_{1}, j_{M-1}\right)} \\
\vdots & \vdots & \vdots & \vdots \\
\mathbf{H}^{\left(i_{N-1}, j_{0}\right)} & \mathbf{H}^{\left(i_{N}-1, j_{1}\right)} & \cdots & \mathbf{H}^{\left(i_{N-1}, j_{M-1}\right)}
\end{array}\right],
$$

where we have:

$$
\begin{aligned}
& 0 \leq i_{0}<i_{1}<\cdots<i_{N-1} \leq N_{r}-1, \\
& 0 \leq j_{0}<j_{1}<\cdots<j_{M-1} \leq N_{t}-1 .
\end{aligned}
$$

Generally speaking, a higher channel gain yields a better system performance. This leads to the joint ApS approach, which selects the specific TAps and RAps related to the particular subset channel matrix associated with the highest channel norm. Since the FSO channels are characterized by real and positive coefficients, the original norm operation applied to $\mathbf{H}^{\left(i_{r}, j_{t}\right)}$ can be omitted, which further reduces the complexity imposed. Hence, the subset channel matrix $\mathbf{H}_{\mathrm{sub}}^{\mathrm{opt}}$ based on the joint ApS criterion is found by solving the optimization problem:

$$
\mathbf{H}_{\mathrm{sub}}^{\mathrm{opt}}=\arg \max _{\mathbf{H}_{\mathrm{sub}} \subset \mathbf{H}} \sum_{t=0}^{M-1} \sum_{r=0}^{N-1}\left\|\mathbf{H}^{\left(i_{r}, j_{t}\right)}\right\|^{2} .
$$

Owing to the specific block-diagonal structure of the full MTRA channel matrix $\mathbf{H}$, the summation in Eq. (22) is over the block-diagonal matrices $\mathbf{H}^{\left(i_{r}, j_{t}\right)}$. Solving the optimization of Eq. (22) by exhaustive search requires the evaluation of the norms of $\left(\mathrm{C}_{N_{r}}^{N} \times \mathrm{C}_{N_{t}}^{M}\right)$ candidate subset matrices, where $\mathrm{C}_{k}^{n}=\frac{k !}{n !(k-n) !}, \mathrm{C}_{N_{r}}^{N}$ and $\mathrm{C}_{N_{t}}^{M}$ are the 'row'-block-dimension and 'column'-block-dimension combinations of $\mathbf{H}_{\mathrm{sub}}$, respectively. By adopting the above-mentioned strategy along with the process to be detailed below in Section III, our joint ApS scheme solves the optimization of Eq. (22) at a significantly reduced complexity.

Given the full channel matrix $\mathbf{H}$ of Eq. (17), without loss of generality, let us assume $C_{N_{r}}^{N}<C_{N_{t}}^{M}$. The joint ApS algorithm accomplishes the optimization in the following two steps.

Step 1): 'Row'-Block Dimension Operations.

Let $n_{r} \in\left\{1,2, \cdots, C_{N_{r}}^{N}\right\}$ be the 'row'-block combination index, and the 'row'-block indices corresponding to the $n_{r}$ th candidate sub-matrix $\mathbf{H}_{n_{r}}$ of $N$ row-blocks and $N_{t}$ column blocks be given by

$$
\boldsymbol{l}_{n_{r}}=\left[l_{n_{r}}^{0} l_{n_{r}}^{1} \cdots l_{n_{r}}^{N-1}\right]^{\mathrm{T}} .
$$

Then the $n_{r}$ th 'row'-block based candidate sub-matrix $\mathbf{H}_{n_{r}}$ is given by

$$
\mathbf{H}_{n_{r}}=\left[\begin{array}{cccc}
\mathbf{H}^{\left(l_{n_{r}}^{0}, 0\right)} & \mathbf{H}^{\left(l_{n_{r}}^{0}, 1\right)} & \ldots & \mathbf{H}^{\left(l_{n_{r}}^{0}, N_{t}-1\right)} \\
\mathbf{H}^{\left(l_{n_{r}}^{1}, 0\right)} & \mathbf{H}^{\left(l_{n_{r}}^{1}, 1\right)} & \ldots & \mathbf{H}^{\left(l_{n_{r}}^{1}, N_{t}-1\right)} \\
\vdots & \vdots & \vdots & \vdots \\
\mathbf{H}^{\left(l_{n_{r}}^{N-1}, 0\right)} & \mathbf{H}^{\left(l_{n_{r}}^{N-1}, 1\right)} & \cdots & \mathbf{H}^{\left(l_{n_{r}}^{N-1}, N_{t}-1\right)}
\end{array}\right] .
$$

Computing

$$
m_{n_{r}}^{x}=\sum_{i=0}^{N-1}\left\|\mathbf{H}^{\left(l_{n_{r}}^{i}, x\right)}\right\|^{2}, 0 \leq x \leq N_{t}-1,
$$

where $m_{n_{r}}^{x}$ represents the square norm of the $x$ th columnblock in $\mathbf{H}_{n_{r}}$, yields the norm metric vector

$$
\mathbf{m}_{n_{r}}^{\mathrm{T}}=\left[m_{n_{r}}^{0} m_{n_{r}}^{1} \cdots m_{n_{r}}^{N_{t}-1}\right] .
$$

Applying Eq. (26) to all the $C_{N_{r}}^{N}$ possible combinations leads to the $\left(\mathrm{C}_{N_{r}}^{N} \times N_{t}\right)$-element norm metric matrix $\mathbf{M}$ given by

$$
\mathbf{M}=\left[\begin{array}{c}
\mathbf{m}_{1}^{\mathrm{T}} \\
\mathbf{m}_{2}^{\mathrm{T}} \\
\vdots \\
\mathbf{m}_{\mathrm{C}_{N_{r}}^{N}}^{\mathrm{T}}
\end{array}\right]=\left[\begin{array}{cccc}
m_{1}^{0} & m_{1}^{1} & \cdots & m_{1}^{N_{t}-1} \\
m_{2}^{0} & m_{2}^{1} & \cdots & m_{2}^{N_{t}-1} \\
\vdots & \vdots & \cdots & \vdots \\
m_{\mathrm{C}_{N_{r}}^{N}}^{0} & m_{\mathrm{C}_{N_{r}}^{N}}^{1} & \cdots & m_{\mathrm{C}_{N_{r}}^{N}}^{N_{t}-1}
\end{array}\right] .
$$

\section{Step 2): 'Column'-Block Dimension Operations.}

Find the largest $M$ elements in the $n_{r}$ th row of $M$ and sum them up, which is denoted as $m_{\max }^{n_{r}}$, as well as record the column-block indices of these $M$ blocks in the index vector

$$
\boldsymbol{l}_{n_{c}}\left(n_{r}\right)=\left[l_{n_{c}}^{0}\left(n_{r}\right) l_{n_{c}}^{1}\left(n_{r}\right) \cdots l_{n_{c}}^{M-1}\left(n_{r}\right)\right]^{\mathrm{T}} .
$$

This produces the max-norm metric vector

$$
\mathbf{m}_{\max }^{\mathrm{T}}=\left[m_{\max }^{1} m_{\max }^{2} \cdots m_{\max }^{\mathrm{C}_{N_{r}}^{N}}\right] .
$$

Next find

$$
\bar{n}_{r}=\arg \max _{1 \leq n_{r} \leq \mathrm{C}_{N_{r}}^{N}} m_{\max }^{n_{r}} .
$$

Then the selected TAp and RAp indices are specified by $l_{n_{c}}\left(\bar{n}_{r}\right)$ and $l_{\bar{n}_{r}}$, respectively, and the corresponding subset channel matrix $\mathbf{H}_{\text {sub }}$ is the optimal solution of Eq. (22). In other words, we have the solution of the optimization problem Eq. (22) given by

$$
\mathbf{H}_{\mathrm{sub}}^{\mathrm{opt}}=\left[\begin{array}{cccc}
\mathbf{H}^{\left(l_{\bar{n}_{r}}^{0}, l_{n_{c}}^{0}\left(\bar{n}_{r}\right)\right)} & \mathbf{H}^{\left(l_{\bar{n}_{r}}^{0}, l_{n_{c}}^{1}\left(\bar{n}_{r}\right)\right)} & \ldots & \mathbf{H}^{\left(l_{\bar{n}_{r}}^{0}, l_{n_{c}}^{M-1}\left(\bar{n}_{r}\right)\right)} \\
\mathbf{H}^{\left(l_{\bar{n}_{r}}^{1}, l_{n_{c}}^{0}\left(\bar{n}_{r}\right)\right)} & \mathbf{H}^{\left(l_{\bar{n}_{r}}^{1}, l_{n_{c}}^{1}\left(\bar{n}_{r}\right)\right)} & \ldots & \mathbf{H}^{\left(l_{\bar{n}_{r}}^{1}, l_{n_{c}}^{M-1}\left(\bar{n}_{r}\right)\right)} \\
\vdots & \vdots & \vdots & \vdots \\
\mathbf{H}^{\left(l_{\bar{n}_{r}}^{N-1}, l_{n_{c}}^{0}\left(\bar{n}_{r}\right)\right)} & \mathbf{H}^{\left(l_{\bar{n}_{r}}^{N-1}, l_{n_{c}}^{1}\left(\bar{n}_{r}\right)\right)} & \cdots & \left.\mathbf{H}^{\left(l_{\bar{n}_{r}}^{N-1}, l_{n_{c}}^{M-1}\left(\bar{n}_{r}\right)\right.}\right)
\end{array}\right] .
$$




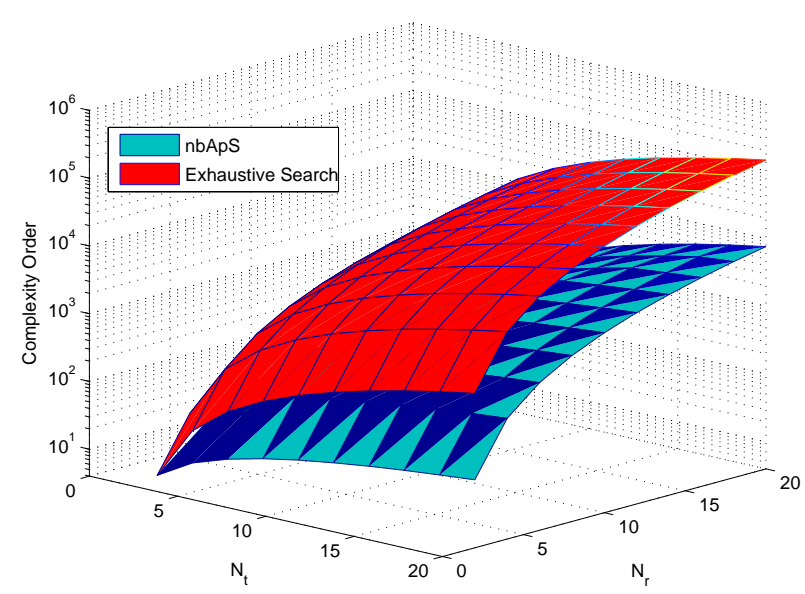

Fig. 2. Complexity comparison of our joint ApS and exhaustive search method, where the number of optical chains at transmit and receive sides are $M=N=2$.

The complexity of this joint $\mathrm{ApS}$ on the order of $C_{\mathrm{nbApS}} \approx$ $\mathcal{O}\left(\left(N_{t} \cdot N\right) \cdot \mathrm{C}_{N_{r}}^{N}\right)$, which is much lower than that of the exhaustive search $C_{\mathrm{ES}} \approx \mathcal{O}\left((N \cdot M) \cdot\left(\mathrm{C}_{N_{t}}^{M} \cdot \mathrm{C}_{N_{r}}^{N}\right)\right)$. If we have $C_{N_{r}}^{N}>C_{N_{t}}^{M}$, the joint ApS starts with Step 1) constituted now by the 'Column'-Block Dimension Operations followed by Step 2), which is now represented by the 'Row'Block Dimension Operations. The complexity of this algorithm is $\mathcal{O}\left(\left(N_{r} \cdot M\right) \cdot \mathrm{C}_{N_{t}}^{M}\right)$. Base on these estimation, the complexity of these two methods is shown in Fig. 2, we may observe that, the complexity of our proposed algorithm is profoundly lower than the complexity obtained from exhaustively search method.

Since $N$ and $M$ are the numbers of available optical chains at the receiver and transmitter, respectively, and since there are the $N_{r} \times N_{t}$ candidate channels, where we have $N_{r}>N$ and $N_{t}>M$, we can define the ApS factor as

$$
f_{A p S}\left(N_{t}, N_{r}\right)=\frac{N_{t}+N_{r}}{M+N},
$$

which determines the 'diversity' order attained by the proposed ACO-OFDM based MTRA system relying on the joint ApS scheme, in comparison with the $(N \times M)$-element ACOOFDM based MTRA system operating without ApS.

\section{Simulation Results}

Simulation based investigations were carried out to evaluate the achievable performance of our ACO-OFDM based MTRA OW systems both without ApS and with the aid of the joint ApS. We simulated the turbulent FSO channel within the physical distance of $L=1000 \mathrm{~m}$ and with the aid of three different refractive index values of $C_{n}^{2}=4 \times 10^{-15}$, $5 \times 10^{-14}$ and $4 \times 10^{-13}$ to represent all weak, moderate and strong turbulence levels, respectively. The modulation schemes employed were $4 \mathrm{QAM}$, and we considered both the G2 and G4 STBC configurations associated with $M=N=2$ and $M=N=4$, respectively, whose system throughput are identical, while the OFDM block length was set to $N_{F}=512$. The signal to noise ratio (SNR) was defined as the ratio of the total transmitted signal power over the total channel noise power.

\section{A. Performance of the Uncoded MTRA System with Joint $M L$-Based HD}

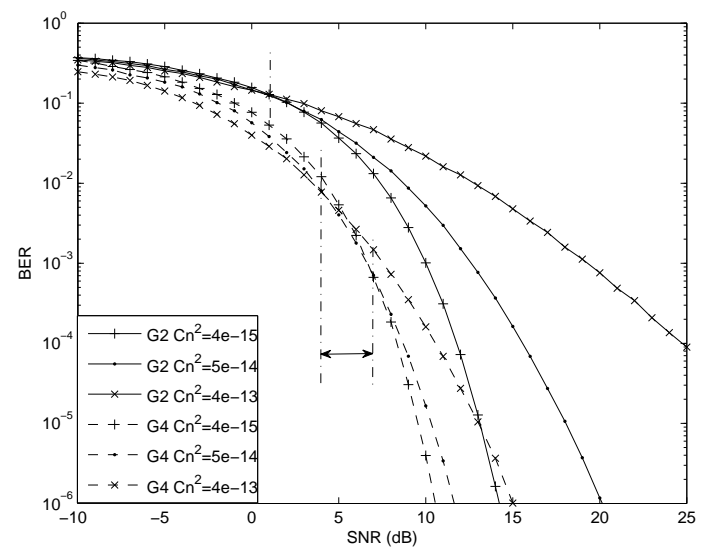

Fig. 3. BER performance of the G2 and G4 STBC based ACOOFDM MTRA systems without ApS, communicating over the FSO turbulence channels of weak, moderate and strong turbulence levels. 'Curve crossing' is marked by dash-dot vertical line.

1) The system without $A p S$ : Let us first consider the basic STBC aided ACO-OFDM MTRA system, where the numbers of TAps and RAps were equal to the numbers of the corresponding transmit and receive optical chains, respectively. Therefore, no ApS was needed. The BER performance of the G2 and G4 STBC based ACO-OFDM MTRA systems operating without ApS are depicted in Fig. 3 for three different channel turbulence levels.

As expected, the system adopting the G4 STBC scheme always outperforms the G2 STBC scheme based system given the same channel turbulence level, since the former attains a higher diversity gain. Intuitively, we would expect that the BER curve of the system operating in a strongly turbulent channel would always be above the BER curve of the same system communicating over a weakly turbulent channel. This trend is similar to that observed for the classic STBC systems in RF communication, where the BER obtained in a high-Doppler-shift environment is typically poorer than that of a low-Doppler-shift environment, unless perfect channel estimation is assumed. However, observe the intriguing BER curve-crossing phenomenon shown in Fig. 3. For example, it can be seen from Fig. 3 that the BER curve of the G4 STBC system operating in the highly turbulent channel associate with $C_{n}^{2}=4 \times 10^{-13}$ is actually lower than that of the same G4 STBC system in the weakly turbulent channel of $C_{n}^{2}=4 \times 10^{-15}$, at $\mathrm{SNR}<5 \mathrm{~dB}$. At SNR $=5 \mathrm{~dB}$, the two BER curves cross over each other, and the performance of the G4 STBC system operating in the strongly turbulent channel becomes poorer than that communicating over the weakly turbulent channel, for SNR $>5 \mathrm{~dB}$. For the G2 STBC scheme, the BER curve cross-over occurs at approximately $\mathrm{SNR}=1 \mathrm{~dB}$, while for the higher-order G4 STBC scheme, the curve-crossing phenomenon is more pronounced and the cross-over takes place in the SNR region of 4 to $7 \mathrm{~dB}$.

The question is then, why this cross-over phenomenon takes place and why it is different from the trends in RF communication. The answer can be found in the comparison we made in Table I between the RF fading channel and the FSO turbulence channel. In practical RF communication, changing the Doppler shift only affects the fading rate of the channel, but the variance of the channel's fading envelop remains the same. Therefore, increasing the Doppler frequency of the fading channel typically degrades the BER performance, unless perfect CSI is assumed. By contrast, 


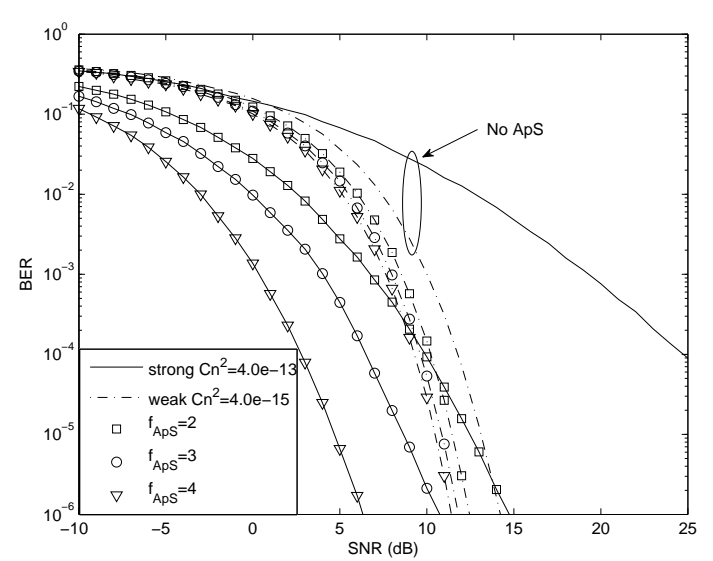

Fig. 4. BER performance enhancement of the G2 STBC based ACO-OFDM MTRA system with the aid of the joint ApS given three different ApS factors over the original G2 STBC based ACO-OFDM MTRA system operating without ApS, communicating over the FSO channels of weak and strong turbulence levels.

the FSO turbulent channel is different from the RF fading channel in this aspect. Firstly, indeed, increasing the channel turbulence's level $C_{n}^{2}$ has a detrimental effect on the BER performance, just as increasing the Doppler frequency will impose a detrimental effect on a $\mathrm{RF}$ fading channel. However, according to Eq. (12), the refractive index $C_{n}^{2}$ also affects the variance of the FSO channel. Thus, changing the turbulence level also affects the channel's PDF. Therefore, unlike in the RF fading channel, the BER curves under different turbulence levels do not start from the same point, and eventually these curves cross over at some SNR point.

2) The system operating with the aid of the joint ApS: We next characterized the BER performance enhancement achieved by the ACO-OFDM MTRA system having $N_{r}>N$ and $N_{t}>M$, which relied on the joint ApS in conjunction with various ApS factors, over the original ACO-OFDM MTRA system, given $N_{r}=N$ and $N_{t}=M$, which operated without ApS. Since the performance enhancements achieved for the G2 and G4 STBC schemes exhibited the same trend, we only showed the corresponding results for the G2 STBC based systems, which are depicted in Fig. 4.

It can be clearly seen from Fig. 4 that the performance enhancement of the G2 STBC based ACO-OFDM MTRA system rely on the joint ApS over the original G2 STBC based ACO-OFDM MTRA system operating without ApS is significantly higher for strongly turbulent channels, than for the weakly turbulent channels. This is because in a weakly turbulent channel, the fluctuations of the CIRs remain low, and the envelope differences among all the candidate aperture pairs are relative small. Therefore, the achievable performance enhancement of $\mathrm{ApS}$ is relatively modest. However, in a high-turbulence channel, the fluctuations of the envelop are more dramatical and the system becomes capable of attaining a much more significant diversity gain. Consequently, more significant performance benefits can be attained by ApS. It can also be observed from Fig. 4 that, for the ApS factors larger than 3, the system's achievable performance under the strongly turbulent channel is actually significantly better than that under weak turbulence. This trend is in stark contrast to that of the original G2 STBC based ACO-OFDM system operating without ApS. Evidently, the performance gain attained by the proposed joint ApS scheme is more significant under strongly turbulent channel

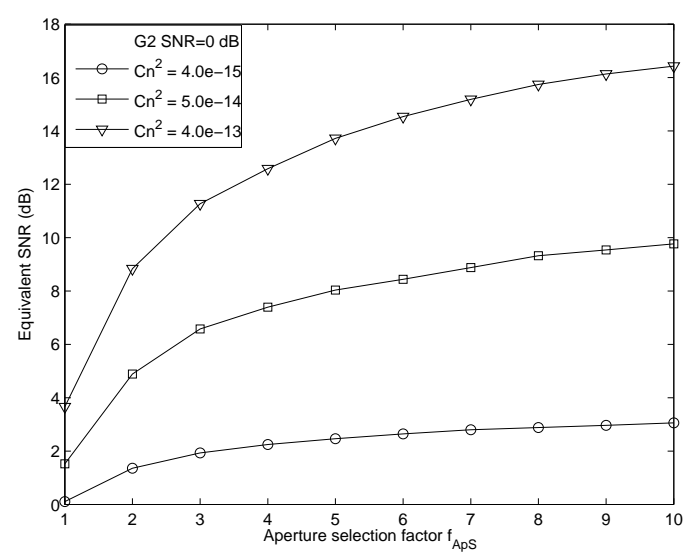

Fig. 5. The equivalent ApS-based SNR as the function of the ApS factor for the G2 STBC based ACO-OFDM MTRA system with the aid of the joint $\mathrm{ApS}$, under different channel turbulent conditions and oiven the SNR in aheones of $A n S$ of $n \mathrm{dR}$

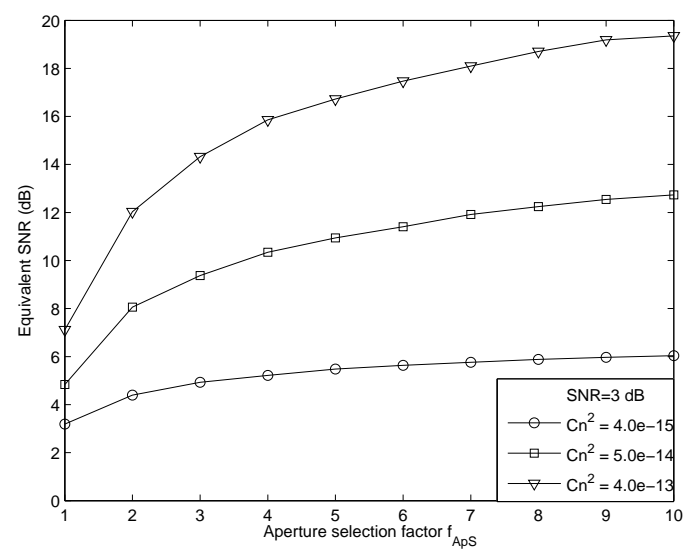

Fig. 6. The equivalent ApS-based SNR as the function of the ApS factor for the G2 STBC based ACO-OFDM MTRA system with the aid of the joint ApS, under different channel turbulent conditions and given the SNR in absence of ApS of $3 \mathrm{~dB}$.

conditions.

3) Equivalent SNR: Intuitively, the diversity gain attained by the joint ApS can be contributed to the carefully selected subset channel matrix of Eq. (31), which contains the subset of the specific CIRs having the highest channel gains. Given the system transmit SNR, let us now define the equivalent ApS-based SNR as

$$
\mathrm{SNR}_{e q u}=10 \log _{10}\left(\frac{\mathrm{SNR}}{M N}\left\|\mathbf{H}_{\mathrm{sub}}^{\mathrm{opt}}\right\|^{2}\right)
$$

Figs. 5 and 6 portray the increased equivalent ApS-based SNRs versus ApS factor $f_{A p S}$ in the three different turbulent channels, given the actual SNRs in absence of ApS of $0 \mathrm{~dB}$ and $3 \mathrm{~dB}$, respectively. It can be seen from Figs. 5 and 6 that for the strongly turbulent channel, the equivalent ApS-based SNR exhibits the most significant improvement owing to ApS, which is correlated by the substantial BER performance improvement shown in Fig. 4. Furthermore, increasing the ApS factor from 1 to 2 provides the highest increase in the equivalent ApS-based SNR. As expected, if we double the transmit power, the corresponding equivalent ApS-based SNR is increased by about $3 \mathrm{~dB}$, as seen by comparing Fig. 5 and Fig. 6. 


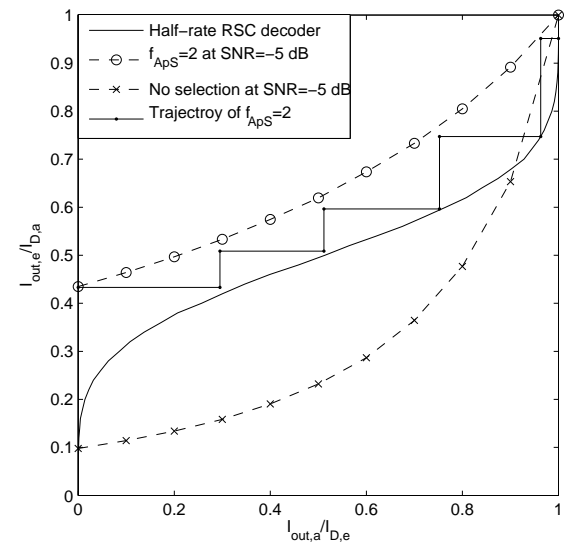

Fig. 7. The EXIT characteristics of the three-stage iteratively decoded G2 STBC based ACO-OFDM MTRA system, communicating over the strongly turbulent channel of $C_{n}^{2}=4 \times 10^{-13}$ and given the SNR of $-5 \mathrm{~dB}$.

\section{B. Performance of the Coded MTRA System with Three-Stage Turbo $S D$ and Decoding}

Next we considered the FEC coded G2 STBC based ACOOFDM MTRA system employing the three-stage iterative joint MAP-based SD and decoding scheme of Fig. 1 presented in Subsection II-C2. In particular, we investigated the beneficial effects of applying the proposed joint ApS scheme to this coded system. In the simulation study, the generator polynomials of the half-rate RSC encoder were expressed in binary format as $G_{R S C}=[1,0,1]_{2}$ and $G_{R S C}^{r}=[1,1,1]_{2}$, while those of the URC encoder were $G_{U R C}=[1,0]_{2}$ and $G_{U R C}^{r}=[1,1]_{2}$, where $G_{R S C}^{r}$ and $G_{U R C}^{r}$ denoted the feedback polynomials of the RSC and URC encoders, respectively. The number of inner iterations and outer iterations were set to $I_{\text {inner }}=2$ and $I_{\text {outer }}=5$. An length of $10^{6}$ bits was used by both of the system interleavers.

1) EXIT chart aided analysis: Having obtain the soft LLRs of the composite inner decoder, let us now invoke EXIT chart for conveniently analyze the convergence behavior by examining the exchange of the input/output mutual information $I_{o u t, a}$ and $I_{o u t, e}$. According to [38], the extrinsic mutual information $I_{o u t, e}$ can be expressed as a function of a priori information $I_{o u t, a}$ and the SNR

$$
I_{\text {out }, e}=\mathbb{T}\left(I_{\text {out }, a}, \mathrm{SNR}\right) .
$$

Furthermore, it has been shown in [38] that the area under the EXIT curve of the composite inner decoder's EXIT chart approximately equals to the system's normalized achievable throughput $\mathcal{A}$, which is directly related to the SNR by [38]:

$$
\mathcal{A}(\mathrm{SNR})=\int_{0}^{1} I_{\text {out }, e} d I_{\text {out }, a}=\int_{0}^{1} \mathbb{T}\left(I_{\text {out }, a}, \mathrm{SNR}\right) d I_{\text {out }, a} .
$$

Thus the overall achievable throughput $\mathcal{T}$ can be written as:

$$
\mathcal{T}(\mathrm{SNR})=\mathcal{A}(\mathrm{SNR}) \cdot M \cdot \log _{2} \zeta .
$$

The a priori and extrinsic mutual information associated with the outer RSC decoder, denoted by $I_{D, a}$ and $I_{D, e}$, can also be similarly calculated.

The EXIT curves of the three-stage coded G2 STBC based ACO-OFDM MTRA system employing the three-stage iterative joint MAP-based SD and decoding scheme communicating over the strongly turbulent channel are depicted

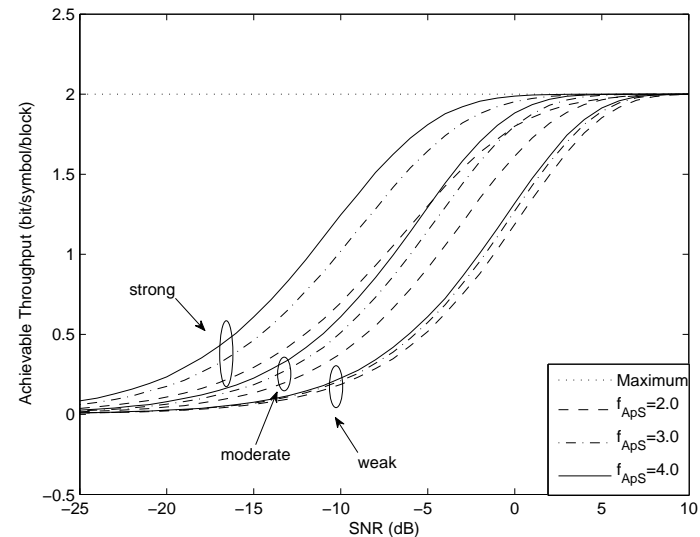

Fig. 8. The achievable throughputs of the three-stage iteratively decoded G2 STBC based ACO-OFDM MTRA system with the aid of the joint ApS scheme having ApS factors of 2, 3 and 4, communicating over different turbulent channels.

in Fig. 7. It can be seen from Fig. 7 that with the aid of the proposed joint ApS scheme and having an ApS factor of 2, an open EXIT-tunnel exists between the EXIT chart curves of the inner SD-URC decoder and the outer RSC decoder at SNR $=-5 \mathrm{~dB}$. The actual stair-case shaped decoding trajectory is also depicted at $\mathrm{SNR}=-5 \mathrm{~dB}$ for this ApS assisted system, which shows that the point of perfect convergence at $(1.0,1.0)$ can be reached after $I_{\text {outer }}=5$ iterations. This indicates that this $\mathrm{ApS}$ assisted system is capable of achieving a vanishingly low $\mathrm{BER}$ at $\mathrm{SNR}=-5 \mathrm{~dB}$, which is confirmed by the system's BER performance shown in Fig. 10. By contrast, the system operating without ApS fails to achieve an open tunnel between the EXIT chart curves of the inner SD-URC decoder and the outer RSC decoder at $\mathrm{SNR}=-5 \mathrm{~dB}$. This implies that the system operating without ApS cannot achieve perfect decoding at $\mathrm{SNR}=-5 \mathrm{~dB}$, which is confirmed by its BER performance shown in Fig. 10.

Additionally, according to Eq. (36), Fig. 8 portrays the achievable throughputs of our proposed ApS assisted system having ApS factors of 2, 3 and 4 and communicating over three different turbulent channels. For this system, the maximum achievable throughput is $0.5 \cdot M \cdot \log _{2} \zeta=$ 2 [bits/symbol/block]. It can be observed from Fig. 8 that for higher ApS factors and for stronger turbulence, a higher throughput is attained for a given SNR value or equivalently a lower SNR is required for reaching a given throughput. The results of Fig. 8 clearly indicate that the performance improvement attained by the proposed joint ApS scheme is much more significant under the strongly turbulent channel of $C_{n}^{2}=4 \times 10^{-13}$, particularly for a large ApS factor of 4 .

2) BER performance: Fig. 9 compares the BER performance of the three-stage iteratively decoded G2 STBC based ACO-OFDM MTRA system relying on the proposed joint ApS scheme having the ApS factors of 2, 3 and 4, respectively, to that of its counterpart operating without ApS, when communicating over the weakly turbulent channel of $C_{n}^{2}=4 \times 10^{-15}$. For the system operating without ApS, the BER convergence of the three-stage iterative decoder is portrayed in Fig. 9, where it can be seen that the system is capable of converging to a vanishing low BER, with the 'turbo-cliff' of the BER curve occurring approximately at $\mathrm{SNR}=2.1 \mathrm{~dB}$ after $I_{\text {outer }}=5$ iterations. For the proposed system assisted by our joint $\mathrm{ApS}$ scheme having the ApS 


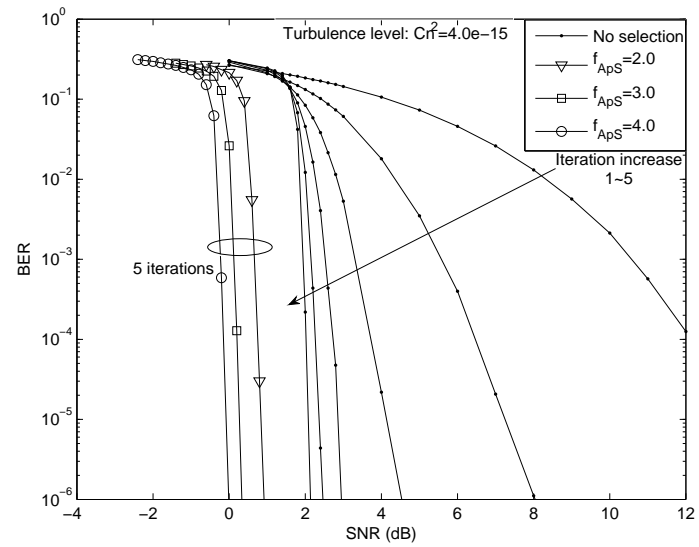

Fig. 9. BER performance comparison of the three-stage iteratively decoded G2 STBC based ACO-OFDM MTRA systems employing the joint ApS scheme having ApS factors of 2, 3 and 4, respectively, as well as operating without ApS, communicating over the weakly turbulent channel.

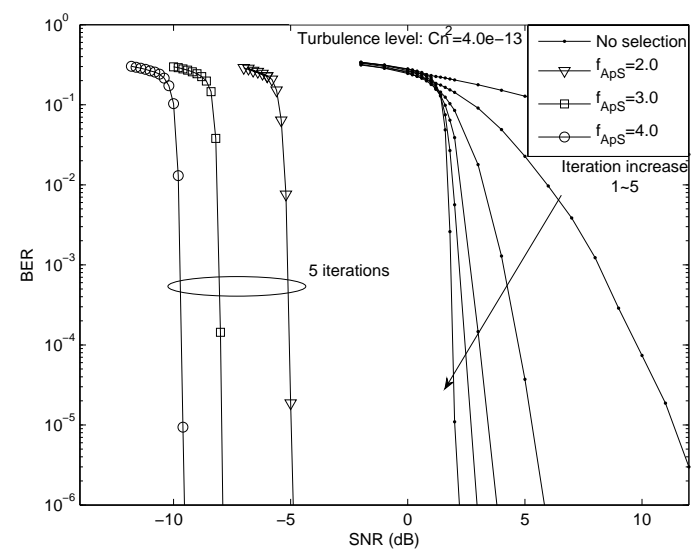

Fig. 10. BER performance comparison of the three-stage iteratively decoded G2 STBC based ACO-OFDM MTRA systems employing the joint ApS scheme having ApS factors of 2, 3 and 4, respectively, as well as operating without ApS, communicating over the strongly turbulent channel.

factors of 2, 3 and 4, by contrast, the turbo-cliff of the BER curve occurs at the SNR values of $0.9 \mathrm{~dB}, 0.3 \mathrm{~dB}$ and $0.0 \mathrm{~dB}$, respectively.

Similarly, Fig. 10 compares the BER performance of the two systems assisted by the joint ApS scheme as well as operating without $\mathrm{ApS}$, when communicating over the strongly turbulent channel of $C_{n}^{2}=4 \times 10^{-13}$. It can be seen from Fig. 10 that for the ApS assisted system having an ApS factor of 2 , a vanishing low $\mathrm{BER}$ is achieved at $\mathrm{SNR}=-5 \mathrm{~dB}$, which matches the prediction by the EXIT chart analysis presented in Fig. 7. When the ApS factor is increased to 3 and 4 , a further performance enhancement is attained and the turbo-cliff of the system's BER curve occurs at the SNR values of $-8 \mathrm{~dB}$ and $-9.7 \mathrm{~dB}$, respectively. By contrast, the system operating without $\mathrm{ApS}$ can only attain a vanishing low BER at approximately SNR $=2 \mathrm{~dB}$. By comparing Fig. 10 and Fig. 9, it can be confirmed again that the performance enhancement achievable by $\mathrm{ApS}$ is much more significant under strongly turbulent channel conditions. Specifically, given the ApS factor of 4, the turbo-cliff of the BER curve occurs at approximately $\mathrm{SNR}=-9.7 \mathrm{~dB}$ under the strongly turbulent level of $C_{n}^{2}=4 \times 10^{-13}$, while it requires $\mathrm{SNR}=$ $0 \mathrm{~dB}$ to attain a vanishingly low BER under the weakly turbulent channel of $C_{n}^{2}=4 \times 10^{-15}$.

\section{Conclusions}

In this paper, we have conceived a joint ApS scheme for a three-stage iteratively decoded ACO-OFDM based MTRA system operates in the FSO turbulence channel. Our main contribution has been the demonstration of such ApS scheme is capable of substantially improving the system's achievable diversity gain, BER performance as well as its throughput, while imposing a moderate hardware complexity. Thus, ApS scheme could enhance the link reliability in V2I and intervehicle communications. Moreover, our results have demonstrated that the performance gain obtained by the novel joint ApS assisted MTRA optical wireless system is more significant under strongly-turbulent channel conditions.

\section{REFERENCES}

[1] V. W. S. Chan, "Free-space optical communications," IEEE J. Lightwave Tech., vol. 24, no. 12, pp. 4750-4762, Dec. 2006.

[2] D. K. Borah and D. G. Voelz, "Pointing error effects on free-space optical communication links in the presence of atmospheric turbulence," IEEE J. Lightwave Tech., vol. 27, no. 18, pp. 39653973, Sept. 2009.

[3] P. Marcsh and G. P. Fettweis, Coordinated multi-point in mobile communications: from theory to practice. Cambridge University Press, 2011.

[4] J. Zhang, R. Zhang, G. Liu and L. Hanzo, "Remote coalition network for base station cooperation aided multicell processing," IEEE Tran. on Veh. Tech., vol. 61, no. 3, pp. 1406-1415, Mar. 2012.

[5] A. Jahn, M. Holzbock, J. Muller, R. Kebel, M. De Sanctis, A. Rogoyski, E. Trachtman, O. Franzrahe, M. Werner, and F. $\mathrm{Hu}$, "Evolution of aeronautical communications for personal multimedia services," IEEE Comm. Mag., vol. 41, no. 7, pp. 3643, July 2003.

[6] Q. Vey, A. Pirovano, J. Radzik, and F. Garcia, "Aeronautical Ad hoc network for civil aviation," in Comm. Tech. for Veh., Springer, 2014, pp. 81-93, 2014.

[7] D. Medina, F. Hoffmann, F. Rossetto, and C.-H. Rokitansky, "Routing in the airborne Internet," in Integrated comm. navi. and surveillance conf. (ICNS) (Herndon, VA), 11-13 May, pp. A71-A7-10, 2010.

[8] D. Medina, Geographic load share routing in the airborne Internet. Herbert Utz Verlag, 2011.

[9] E. Haas, "Aeronautical channel modeling," IEEE Tran. on Veh. Tech., vol. 51, no. 2, pp. 254-264, Mar. 2012.

[10] J. Haque, An OFDM based aeronautical communication system. $\mathrm{Ph} . \mathrm{D}$ disertation, University of South Florida, 2011.

[11] J. Kuchar and A. C. Drumm, "The traffic alert and collision avoidance system," Lincoln Lab. J., vol. 16, no. 2, pp. 277-296, 2007.

[12] E. Williams, "Airborne collision avoidance system," in SCS, vol. 4, pp. 87-110, 2004.

[13] EUROCONTROL. "Airborne seperation assurance systems (ASAS)," [Online]. Available: http://www.skybrary.aero/index.php/Airborne_Separation Assurance_Systems_(ASAS)

[14] S. Bretmersky, V. K. Konangi and R. J. Kerczewski, "Comparison of vdl modes in the aeronautical telecommunications network," in IEEE Aero. Con. Proc., vol. 3, pp. 3-1183-3-1190, 2002.

[15] S. Nicol, G. Wlton, L. Westbrook, and D. Wynn, "Future satellite communication to military aircraft," Elec. \& Comm. Engineering J., vol. 12, no. 1, pp. 15-26, 2000.

[16] G. Berzins, R. Phillips, J. Singh, and P. Wood, "Inmarsatworldwide mobile satellite services on seas, in air and on land," in Malaga Inter. Astron. Fed. Cong. vol. 1, 1989.

[17] E. J. Lee and V. W. S. Chan, "Part 1: Optical communication over the clear turbulent atmospheric channel using diversity," IEEE J. S. A. in Comm., vol. 22, no. 9, pp. 1896-1906, Nov. 2004.

[18] J. Armstrong and A. J. Lowery, "Power efficient optical OFDM," Elect. Letters, vol. 42, no. 6, pp. 370-372, Mar. 2006. 
[19] W. Xu, M. Wu, H. Zhang, X. You, and C. Zhao, ACO-OFDM specified recoverable upper-clipping with efficient detection for optical wireless communications, IEEE Photon. J., Oct. 2014.

[20] B. Ranjha and M. Kavehrad, Hybrid asymmetrically clipped OFDM based IM/DD optical wireless system, IEEE J. Opt. Comm. Netw., vol. 6, no. 4, pp. 387396, Apr. 2014.

[21] N. Fernando, H. Yi and E. Viterbo "Flip-OFDM for Unipolar Communication Systems," IEEE Trans. on Comm., vol. 60, no. 12 , pp. $3726-3733$, Dec. 2012.

[22] I.B. Djordjevic, B. Vasic and M. A. Neifeld, "LDPC-COded OFDM for Optical Communication Systems with Direct Detection," IEEE Trans. S. Top. in Quan. Elec., vol. 13, no. 5, pp. 14461454, Sept. 2007.

[23] I.B. Djordjevic and H. G. Batshon, "LDPC-Coded OFDM for Heterogeneous Access Optical Networks," IEEE Photon. J., vol. 2, no. 4, pp. 611-619, Aug. 2010.

[24] C. Chen, W. D. Zhong, X. Li and D. Wu, "MDPSK-Based Nonequalization OFDM for Coherent Free-Space Optical Communication," IEEE Photon. Tech. Letter, vol. 26, no. 16, pp. 16171620, June 2014.

[25] S. Sanayei and A. Norsratinia, "Antenna selection in MIMO systems," IEEE Comm. Mag., vol. 42, no. 10, pp. 68-73, Oct. 2004.

[26] T. Gucluoglu and T. M. Duman, "Performance analysis of transmit and receive antenna selection over flat fading channels," IEEE Trans. Wirel. Comm., vol. 7, no. 8, pp. 3056-3065, Aug. 2008.

[27] M. Gharavi-Alkhansari and A. B. Gershman, "Fast antenna subset selection in MIMO systems," IEEE Trans. Sig. Proc., vol. 52, no. 2, pp. 339-347, Feb. 2004.

[28] A. G. Zambrana, C. C. Vázquez, , B. C. Vázquez and A. H. Gómez, "Selection transmit diversity for FSO links over strong atmospheric turbulence channels," IEEE Photon. Tech. Letters, vol. 21, no. 14, pp. 1017-1019, May 2009.

[29] A. Sevincer, Transceiver selection for multi-element freespace-optical communications . Ph.D disertation, University of Nevada, 2013.

[30] H. Moradi, H. H. Refai, and P. G. LoPresti, "Circular MIMO nodes with transmit selection and receive generalized selection diversity," IEEE Tran. on Veh. Tech., vol. 61, no. 3, pp. 15831589, Mar. 2012.

[31] N. D. Chatzidiamantis, D. S. Michalopoulos, E. E. Kriezis, G. K. Karagiannidis, and R. Schober, "Relay selection protocols for relay-assisted free-space optical systems," J. Opt. Comm. Netw., vol. 5, no. 1, pp. 92-103, Jan. 2013.

[32] A. Goldsmith, Wireless Communications. Cambridge University Press, 2005.

[33] M. A. Al-Habash, L. C. Andrews, and R. L. Phillips, "Mathematical model for the irradiance probability density function of a laser beam propagating through turbulence media," Opt. Engineering, vol. 40, no. 8, pp. 1554-1562, Aug. 2001.

[34] J. Armstrong, "Alamouti coding for indoor optical wireless communications using ACO-OFDM," Asilomar Conf. on Sig., Sys. and Com., pp. 1650-1654, Nov. 2009.

[35] M. El-Hajjar and L. Hanzo, "EXIT charts for system design and analysis," IEEE Comm. Sur. and Tutor., vol. 16, no. 1, pp. 127$153,2014$.

[36] J. Wang, S. X. Ng, A. Wolfgang, L. L. Yang, S. Chen, and L. Hanzo, "Near-capacity three-stage MMSE turbo equalization using irregular convolutional codes," in: Proc. Turbo-Coding2006 (Munich, Germany), April 3-7, 2006, 6 pages.

[37] R. G. Maunder and L. Hanzo, "Iterative decoding convergence and termination of serially concatenated codes," IEEE Trans. Veh. Tech., vol. 59, no. 1, pp. 216-224, Jan. 2010.

[38] A. Ashikhmin, G. Kramer, and S. ten Brink, "Extrinsic information transfer functions: Model and erasure channel properties," IEEE Trans. Info. Theo., vol. 50, no. 11, pp. 2657-2673, Nov. 2004 .

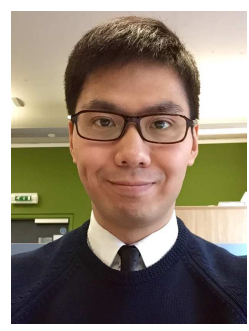

Junyi Jiang received his BEng degree in communication engineering from Heilongjiang Institute of Science and Technology, PRC, in 2009 and his MSc degree with distinction in wireless communication from the University of Southampton, UK, in 2010. He is currently working towards the $\mathrm{PhD}$ degree with the Wireless Group, the University of Southampton, UK.

His research interests include indoor visible light communication, free-space optical communication and iterative detection.

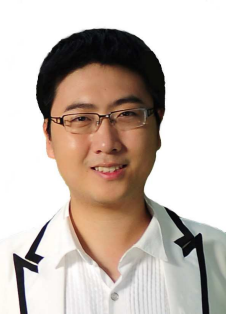

Peichang Zhang received the B.Eng. degree (with first-class honors) in electronic engineering from the University of Central Lancashire, Lancashire, U.K., in 2009 and the M.Sc. degree with distinction in wireless Communications from the University of Southampton, Southampton, U.K., in 2010. He has received Ph.D. degree with the Communications Signal Processing, and Control Research Group, Department of Electronics and Computer Science, University of Southampton. He is now holding the lecturing position in the department of CIE, Shenzhen University, China. His research interests include coherent and non-coherent detection, iterative detection and estimation

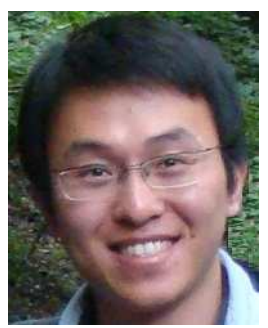

Rong Zhang (M'09) received his PhD (Jun 09) from Southampton University, UK and his BSc (Jun 03) from Southeast University, China. Before doctorate, he was an engineer (Aug 03-July 04) at China Telecom and a research assistant (Jan 06-May 09) at Mobile Virtual Center of Excellence (MVCE), UK. After being a post-doctoral researcher (Aug 09July 12) at Southampton University, he took industrial consulting leave (Aug 12-Jan 13) for Huawei Sweden R\& D as a system algorithms specialist. Since Feb 13, he has been appointed as a lecturer at Southampton Wireless group of ECS, Southampton University. He has $40+$ journals in prestigious publication avenues (e.g. IEEE, OSA) and many more in major conference proceedings. He regularly serves as reviewer for IEEE transactions/journals and has been several times as TPC member/invited session chair of major conferences. He is the recipient of joint funding of MVCE and EPSRC and is also a visiting researcher under Worldwide University Network (WUN). More details can be found at http://www.ecs.soton.ac.uk/people/rz 


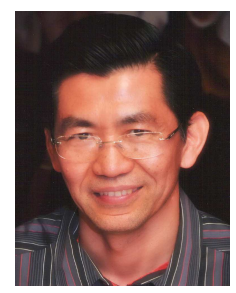

Sheng Chen (M'90-SM'97-F'08) received his BEng degree from the East China Petroleum Institute, Dongying, China, in 1982, and his PhD degree from the City University, London, in 1986, both in control engineering. In 2005, he was awarded the higher doctoral degree, Doctor of Sciences (DSc), from the University of Southampton, Southampton, UK.

From 1986 to 1999 , He held research and academic appointments at the Universities of Sheffield, Edinburgh and Portsmouth, all in UK. Since 1999, he has been with Electronics and Computer Science, the University of Southampton, UK, where he holds the post of Professor in Intelligent Systems and Signal Processing. Dr Chen's research interests include adaptive signal processing, wireless communications, modelling and identification of nonlinear systems, neural network and machine learning, intelligent control system design, evolutionary computation methods and optimisation. He has published over 550 research papers.

Dr. Chen is a Fellow of IET, a Distinguished Adjunct Professor at King Abdulaziz University, Jeddah, Saudi Arabia, and an ISI highly cited researcher in engineering (March 2004). He was elected to a Fellow of the United Kingdom Royal Academy of Engineering in 2014.

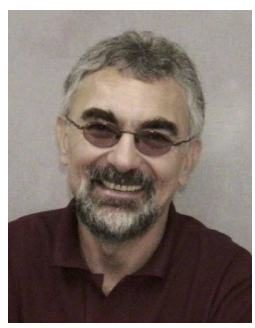

Lajos Hanzo FREng, FIEEE, FIET, Fellow of EURASIP, DSc received his degree in electronics in 1976 and his doctorate in 1983. In 2009 he was awarded the honorary doctorate "Doctor Honoris Causa" by the Technical University of Budapest. During his 38-year career in telecommunications he has held various research and academic posts in Hungary, Germany and the UK. Since 1986 he has been with the School of Electronics and Computer Science, University of Southampton, UK, where he holds the chair in telecommunications. He has successfully supervised about $100 \mathrm{PhD}$ students, co-authored 20 John Wiley/IEEE Press books on mobile radio communications totalling in excess of 10000 pages, published 1500 research entries at IEEE Xplore, acted both as TPC and General Chair of IEEE conferences, presented keynote lectures and has been awarded a number of distinctions. Currently he is directing a 100-strong academic research team, working on a range of research projects in the field of wireless multimedia communications sponsored by industry, the Engineering and Physical Sciences Research Council (EPSRC) UK, the European Research Council's Advanced Fellow Grant and the Royal Society's Wolfson Research Merit Award. He is an enthusiastic supporter of industrial and academic liaison and he offers a range of industrial courses. He is also a Governor of the IEEE VTS. During 2008 - 2012 he was the Editor-in-Chief of the IEEE Press and a Chaired Professor also at Tsinghua University, Beijing. His research is funded by the European Research Council's Senior Research Fellow Grant. For further information on research in progress and associated publications please refer to http://wwwmobile.ecs.soton.ac.uk Lajos has $22000+$ citations. 